\title{
Redes sociales online y su utilización para mejorar las habilidades sociales en jóvenes con discapacidad
}

\section{Online social networks and using them to improve social skills in young people with disabilities}

\author{
Raquel Suriá Martínez \\ Departamento de Comunicación y Psicología Social. Universidad de Alicante. España \\ Disponible online 31 de diciembre de 2012
}

\begin{abstract}
En los últimos años, las redes sociales online han introducido profundos cambios en nuestro entorno y en los modos de relacionarnos con los demás. Esto, que es frecuente en multitud de jóvenes, puede potenciarse más en los jóvenes con movilidad reducida derivada de una discapacidad. En este trabajo se examina la opinión que tienen los jóvenes con discapacidad motora sobre las relaciones de amistad que mantienen a través de las redes online así como la percepción sobre éstas para mejorar sus habilidades sociales. Esto se analiza en función del género, edad y ocupación. Han participado 68 jóvenes con discapacidad motora que contestaron un cuestionario online, creado para tal fin. Los resultados se analizaron a través de una metodología cuantitativa. Aunque se encontraron diferencias significativas en función del género, edad y ocupación, en la mayoría de jóvenes se percibe una alta motivación en el uso de estos espacios para sus relaciones personales. Asimismo, valoran las redes para mejorar sus habilidades sociales. Ello sugiere la importancia que tienen para los jóvenes con discapacidad física las redes sociales online en sus relaciones sociales, a la vez que podrían servir como ayuda en los programas dirigidos a fomentar la integración social.
\end{abstract}

Palabras clave: Jóvenes; Discapacidad; Redes sociales online; Habilidades sociales.

In recent years, online social networks have introduced profound changes in our environment and new ways of relating to others. The use of social networks is increasingly common, especially among young people, and could further empower young people with limited mobility as a result of a disability. This paper examines the opinions of young people with motor disabilities about the friendships they maintain over online networks and their perceptions regarding how these networks improve their social skills. This is discussed in reference to gender, age and occupation. 68 young people with physical disabilities answered an online questionnaire created for this purpose. The results were analyzed using quantitative methodology. Although we found significant differences based on gender, age and occupation, most of the young people perceived themselves as being highly motivated to use these spaces for personal relationships. They also value the networks in improving their social skills. These results suggest that online social networks are important to young people with physical disabilities in their social relationships, and may also help to design programs aimed at promoting social integration.

Key words: Young people; Disability; Social networking online; Social skills.

Correspondencia: Raquel Suriá Martínez. Universidad de Alicante, Dpto. Comunicación y Psicología Social. Apdo. Correos, 99. Campus de San Vicente del Raspeig - 03080 Alicante. E-mail: raquel.suria@ua.es 
En los últimos años, la rápida evolución de internet ha cambiado los hábitos de la sociedad y las redes sociales se han convertido en una nueva vía en la que crear espacios de interacción para muchas personas (Bernete, 2010; Buckingham, 2008; Lenhart, Madden, Rankin Macgill y Smith, 2007; Livingstone, 2008; Rubio-Gil, 2010).

Entre las características que motivan la expansión de estos espacios, podríamos destacar la facilidad para acceder a cualquier hora del día, los bajos costes, la respuesta rápida y las recompensas inmediatas, particularidades que hacen de estos espacios virtuales unos sitios muy atractivos para diferentes colectivos, siendo los jóvenes y adolescentes los usuarios que más utilizan las redes online para interaccionar socialmente (Aguaded, 2009; Livingstone y Helsper, 2010; Yang y Tung, 2007). Así, por ejemplo los datos el último informe realizado por la Asociación para la Investigación de los Medios de Comunicación (AIMC, 2011) indican que, en España, alrededor del $80 \%$ de los jóvenes utiliza estos espacios para interaccionar, siendo la franja de edad más importante entre los 25-34 años.

Del mismo modo, otras particularidades de esta forma de interacción como la facilidad para expresarse más libremente que en ambientes cara a cara, el acceso a una cantidad de información más heterogénea y variada, el anonimato, o no tener que desplazarse para comunicarse son sin duda, aspectos que para muchos usuarios favorecen su uso (Finn, 1999; Suriá y Beléndez, 2011; Weinberg, Schmale, Uken y Wessel, 1995; Wright, 2002; Wright y Bell, 2003).

Sin embargo, estas ventajas que son evidentes para los usuarios pueden verse potenciadas en mayor medida en algunos colectivos (Alexander, Peterson y Hollingshead, 2003; Finn, 1999; Fogel, Albert, Schnabel, Ditkoff y Neugut, 2002; Hoybye, Johansen y Tjornhoj-Thomsen, 2005), como son los jóvenes que tienen movilidad reducida como consecuencia de una discapacidad motora.

En referencia a este colectivo, en España, según los datos de la Encuesta sobre Discapacidad, Autonomía personal y situaciones de Dependencia (EDAD), realizada por el Instituto Nacional de Estadística (2008), se estima que viven 163.650 personas con discapacidad entre 15 y 30 años, de estas cifras, un alto porcentaje de estos jóvenes (52\%) tienen discapacidad motora que, bien porque han adquirido una discapacidad en el nacimiento (espina bífida, parálisis cerebral, distrofia muscular, etc.) o porque les ha sobrevenido la discapacidad a lo largo de la vida (lesión medular por accidente de tráfico, enfermedad degenerativa, etc...), tienen limitaciones en su movilidad.

Estas limitaciones derivan en que pueda verse reducida su vida social y con ello, sus relaciones sociales tradicionales o cara a cara. Sin embargo, la red puede beneficiarles al eliminar las barreras geográficas, e incluso en algunos casos, a anular o mitigar las barreras estigmatizantes derivadas de su discapacidad, pudiendo de este modo, llegar a ser espacios de utilidad no únicamente porque sea más fácil crear y mantener contactos, sino también por ser plataformas en las que se creen comunida- des o grupos de apoyo para compartir experiencias más libremente con otros usuarios.

No obstante, aunque la mayor parte de la literatura sobre las redes sociales se ha dirigido a conocer las potencialidades de estos espacios virtuales, destacando las anteriormente citadas como positivas, otros estudios sugieren que algunas de ellas pueden ser perjudiciales. Por ejemplo, la ausencia de señales visuales y de otras señales no verbales, que normalmente acompañan a las interacciones cara a cara. De éstas se extrae la información del interlocutor, sin embargo, a través de la pantalla se pierde esta información. Del mismo modo, el anonimato puede encubrir a usuarios que se dedican a amenazar, insultar o al fenómeno desarrollado en estos últimos años, el acoso por internet o "grooming".

Otra de las críticas que más ha proliferado ente otros autores son los posibles efectos negativos de su utilización como es la vinculación del uso de internet con el aislamiento social. Por ejemplo, numerosos estudios han investigado la relación existente entre el uso de internet y la soledad, encontrando una asociación positiva (Beranuy, Lusar, Jordania y Sanchez, 2009; Cruzado, Matos y Kendall, 2006; Echeburúa y de Corral, 2009; García del Castillo et al, 2007; Kraut, Patterson, Landmark, Kiesler, Mukophadhyay y Scherlis, 1998; Morahan - Martin y Schumacher, 2003; Poch, 2009; Young, 2009). Sin embargo, los datos que explican estos resultados son contradictorios. Así, se han propuesto dos hipótesis alternativas: el uso excesivo de internet causa soledad o, por el contrario, son las personas que se sienten solas las más propensas a abusar de internet (Morahan-Martin y Schumacher, 2003).

La primera hipótesis surgió de un estudio llevado a cabo por Kraut et al. (1998). En sus resultados encontraron en un grupo de usuarios participantes que después de dos años de comunicarse a través de internet, existía una menor participación social (comunicación familiar, red social y apoyo social) y una disminución del bienestar psicológico (aumento de la soledad y de depresión).

A diferencia de estos resultados, otros estudios no encontraron ningún declive en las relaciones sociales ni en la comunicación que mantenían cara a cara con la familia, amigos o compañeros de trabajo (Robinson, Kestnbaum, Neustadtl y Alvarez, 2000; UCLA Centro para la Política de Comunicación, 2000). Al mismo tiempo, otros autores (Fowler y Christakis, 2009; Zubeidat, Sierra y Salinas, 2007) han encontrado en sus resultados que ese uso de internet no sólo mejora la interacción y existencia de relaciones sociales, sino que incluso aumenta la cantidad de conexiones en la red social.

Ante los resultados ofrecidos por las anteriores hipótesis y que, como en líneas anteriores se ha mencionado, con frecuencia, las personas con discapacidad motora ven reducida su red social debido a las limitaciones de movilidad con la que conviven, ¿son usuarios los jóvenes con esta problemática de las redes sociales online?, si esto es así, ¿conectan con asiduidad para mantener contacto con sus amigos?, ¿las utilizan para 
conectar con amigos tradicionales? o por el contrario, ¿entran para interaccionar con amigos conocidos en la red?

En este sentido, autores como Mickelson (1997) citado en Kiesler (2001), sugiere que algunos usuarios encuentran en la red a otros usuarios con los que compartir experiencias que no compartirían con personas cara a cara.

De este modo y siguiendo la idea de este autor, nos encontramos que estas redes posibilitan en primer lugar, disminuir las distancias geográficas y comunicarnos con conocidos de la vida real, a su vez, permiten interactuar con otros usuarios de la red con los que se prefiere tener una comunicación exclusivamente virtual, al tiempo que, estas relaciones pueden transcender y llegar a convertirse en relaciones tradicionales y no ser únicamente conexiones a través de la red (Del Moral, 2007; Tully, 2007; Young, 2009).

A su vez, cabría la posibilidad de que el establecimiento de relaciones con desconocidos a través de estos espacios online, de algún modo, pudiera resultar enriquecedor no sólo por ampliar la red social sino porque al fomentar la interacción social facilitara el desarrollo de habilidades sociales de la persona. Por ejemplo, expresase con mayor libertad, el feedback proporcionado por los otros usuarios ante el mensaje del que escribe, o la búsqueda y perfeccionamiento de formas de expresión personal son algunas de las habilidades sociales que pueden fomentarse a través de las redes sociales. Ante esto, ¿podría ser posible que los usuarios más jóvenes encontraran en estos espacios unos sitios atractivos para fomentar sus habilidades sociales?

A partir de lo anterior en este estudio pretendemos conocer la opinión que tienen los jóvenes con discapacidad motora sobre el uso de las redes sociales como espacios para relacionarse. Esto se analiza en función del género, de la edad y de la ocupación de estos jóvenes. Este objetivo general se desglosa en los siguientes objetivos específicos:

- Conocer el perfil de utilización que hacen los jóvenes con discapacidad motora de estas redes.

- Averiguar en qué medida utilizan estos espacios para relacionarse con su red social tradicional o para relacionarse con amigos conocidos en la red.

- Conocer si a través de estos espacios han ampliado su red social.

- Finalmente, indagar sobre la percepción que tienen de que las redes online les hayan ayudado a incrementar sus habilidades sociales.

Método
Sujetos
En este estudio participaron 68 jóvenes con discapacidad
motora ( $42.6 \%$ mujeres y $57.4 \%$ varones), con edades com-
prendidas entre los 18 y 35 años, los cuales fueron divididos
en dos grupos según la edad (entre 18 y 25 y entre 26 y 35$)$.
Establecimos los 25 años de edad de corte pensando en que
es a partir de esa edad, en la que por término medio, un joven

finaliza los estudios y se incorpora al mudo laboral y con ello, a su independencia y adultez). Con respecto a la ocupación, el $33.8 \%$ eran estudiantes, el $19.1 \%$ estaban en situación laboral activa y el $47.1 \%$ indicó que no estaba ni estudiando ni trabajando en ese momento.

\section{Instrumentos}

El instrumento empleado ha sido un cuestionario elaborado y validado para el estudio, el cual está formado por dos bloques. Dicho instrumento está formado por un bloque de cuestiones sociodemográficas. La segunda parte del instrumento consta de una escala tipo Likert de cinco puntos, desde $1=$ nada a $5=$ mucho), enfocado a conocer la frecuencia de uso de las redes online y sobre la utilización que hacen de las redes sociales en sus relaciones sociales.

Para cumplir con los requisitos de validez de contenido se aplicó el protocolo de validación de contenido, que determina la relevancia o representatividad de los ítems en relación a la muestra establecida (Latiesa, 1996; Losada y López-Feal, 2003). Para ello, 2 jueces expertos respondieron a un cuestionario dicotómico que indagaba sobre la validez o no de cada ítem propuesto, a los resultados obtenidos se aplicó la Distribución Binomial para cada ítem, quedando el cuestionario, que en un principio estaba formado por 12 ítems, reducido a 10 ítems. En relación a la validez operativa, los mismos expertos efectuaron recomendaciones en función de las cuales se ajustaron las preguntas. La fiabilidad del cuestionario se determinó mediante el Coeficiente Alfa de Cronbach, obteniendo un índice de consistencia interna de 0.76 .

\section{Procedimiento}

Una vez diseñado el cuestionario, a través de la empresa "Google" se creó un apartado específicamente para la línea de investigación a la que se accede a través de un enlace alojado en Google y en el que se pedía la colaboración de las personas con discapacidad motora. Para dar a conocer el enlace entramos en un espacio virtual dedicado al ámbito de la discapacidad, disponible en Facebook destinado a temas de accesibilidad universal desarrollado por el observatorio de la discapacidad del Centro de apoyo al estudiante de la universidad de Alicante. A su vez, colocamos el enlace en algunos espacios de la red social Facebook y Twitter creados por diferentes asociaciones dirigidas a personas con discapacidad motora en los que pusimos un mensaje explicando brevemente nuestro objetivo, solicitando su colaboración para rellenar el cuestionario ubicado en el enlace inscrito en el mensaje. Finalmente, los investigadores tenían vinculación directa con algunos jóvenes con discapacidad motora, con éstos, se utilizó el muestreo "bola de nieve", dando a conocer primero el enlace y posteriormente, se les solicitó que dieran a conocer el cuestionario a otros jóvenes con discapacidad motora. A continuación, se procedió al proceso de recogida de datos. La recopilación de los cuestionarios se llevó a cabo durante 1 mes. 


\section{Análisis de datos}

Para conocer el perfil de uso de las redes online de los jóvenes con discapacidad se hallaron las frecuencias y porcentajes de las variables sociodemográficas. Para hallar las comparaciones entre los participantes en el uso y satisfacción de las redes sociales virtuales se utilizó la prueba no paramétrica $U$ de Mann-Whitney en las variables de 2 grupos (edad y género) y la prueba de Kruskall-Wallis en la variable de más de 2 grupos (ocupación).

\section{Resultados}

En primer lugar, al examinar el perfil de uso de los participantes encontramos (Tabla 1), que el 50\% afirmó conectar varias veces al día, observándose que la mayoría le dedican de 2 a 4 horas (45.68\%) a estos espacios siendo el motivo principal para el que los utilizan es por amistad $(83.8 \%)$ a la vez que indicaron alrededor del $47.1 \%$ tener ente 21 y 50 amigos en la red.

Con respecto a la utilización que los jóvenes con discapacidad hacen de las redes sociales online para relacionarse sus amistades encontramos que un $29.41 \%$ indicó estar bastante de acuerdo en utilizar esos espacios para relacionarte con amigos tradicionales.

Este porcentaje fue más elevado al referirnos en cuanto a la utilización para relacionarse con amigos que han conocido en la red, en el que el $35.29 \%$ afirmo estar bastante de acuerdo.

En lo concerniente a la calidad de las relaciones que mantienen a través de las redes online observamos que el $25 \%$ mostró estar muy de acuerdo y (25\%) bastante de acuerdo en considerar que estas relaciones virtuales son igual de importantes que las relaciones tradicionales.

Asimismo, el 38.24\% indicó estar bastante de acuerdo en afirmar que desde que entra en estos espacios ha aumentado el número de amigos que tienen. Por último, los datos globales sobre la percepción que tienen los participantes de que es un recurso de ayuda para mejorar las habilidades sociales observamos que el $41.18 \%$ estuvo muy de acuerdo en afirmar que las redes fomentan las habilidades sociales a la vez que el $32.35 \%$ consideró que el uso de estas redes les ha ayudado a mejorar sus habilidades sociales.

En cuanto a los interrogantes planteados sobre la utilidad de las redes en función de las variables analizadas, esto es, del género, edad y ocupación de los participantes pueden observarse los resultados en la tabla 3.

Con respecto al género, observamos que tanto varones como mujeres utilizan estos espacios para relacionarse con los amigos tradicionales, $U(1)=560.5, p=.949$. Sin embargo, sí se observan diferencias estadísticamente significativas en el uso de estas redes para relacionarse con amigos conocidos a través de la red, apreciándose que las mujeres indicaron mayor referencia por esto, $U(1)=335, p<.05$.

Del mismo modo, indicaron estar más de acuerdo en considerar igual de importantes los amigos virtuales que los tradicionales, $U(1)=387, p<.05$.

Por otra parte, no se observaron diferencias a nivel estadísticamente significativo en cuanto a tener más amigos desde que

Tabla 1

Perfil de uso de las redes sociales. Frecuencias y tantos por ciento.

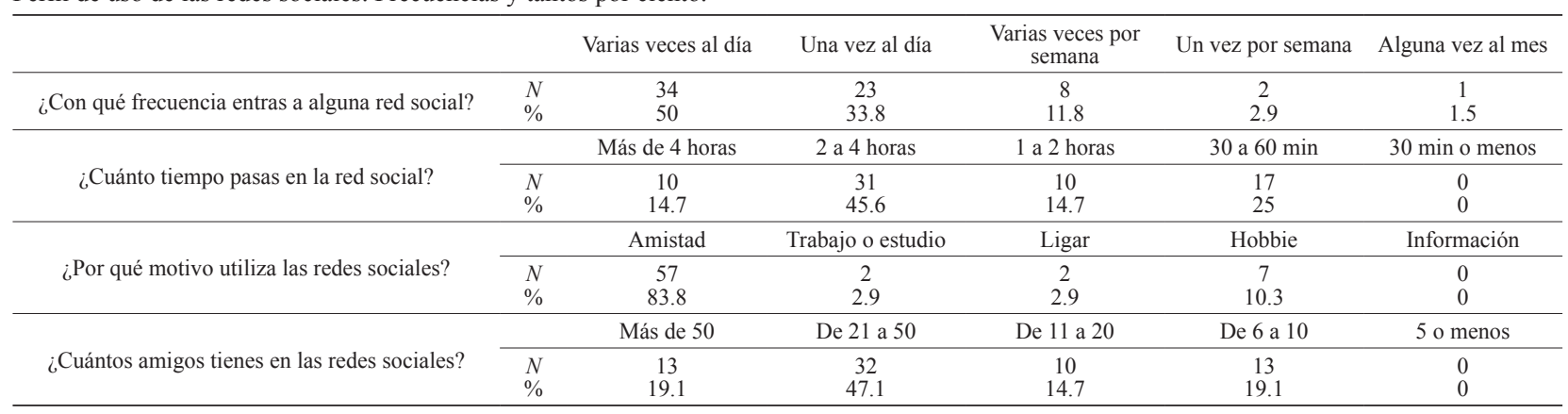

Tabla 2

Utilidad de las redes sociales online. Frecuencias y tantos por ciento.

\begin{tabular}{|c|c|c|c|c|c|c|}
\hline Utilidad de las redes sociales online & & Nada & Poco & Algo & Bastante & Mucho \\
\hline ¿Utilizas las redes para relacionarte con amigos tradicionales? & $\begin{array}{l}N \\
\%\end{array}$ & $\begin{array}{c}10 \\
14.70\end{array}$ & $\begin{array}{c}17 \\
25.00\end{array}$ & $\begin{array}{c}11 \\
16.18\end{array}$ & $\begin{array}{c}20 \\
29.41\end{array}$ & $\begin{array}{c}10 \\
14.71\end{array}$ \\
\hline ¿Utilizas las redes para relacionarte con amigos conocidos de la red? & $\begin{array}{l}N \\
\%\end{array}$ & $\begin{array}{c}4 \\
5.88\end{array}$ & $\begin{array}{c}8 \\
11.76\end{array}$ & $\begin{array}{c}23 \\
33.82\end{array}$ & $\begin{array}{c}24 \\
35.29\end{array}$ & $\begin{array}{c}9 \\
13.24\end{array}$ \\
\hline ¿Tienes más amigos desde que entras a las redes? & $\begin{array}{l}N \\
\%\end{array}$ & $\stackrel{5}{7.35}$ & $\begin{array}{c}18 \\
26.47\end{array}$ & $\begin{array}{c}14 \\
20.59\end{array}$ & $\begin{array}{c}26 \\
38.24\end{array}$ & $\begin{array}{c}5 \\
7.35\end{array}$ \\
\hline ¿Consideras igual de importantes tus amigos virtuales que los tradicionales? & $\begin{array}{l}N \\
\%\end{array}$ & $\begin{array}{c}11 \\
16.17\end{array}$ & $\begin{array}{c}15 \\
22.06\end{array}$ & $\begin{array}{c}8 \\
11.76\end{array}$ & $\begin{array}{c}17 \\
25.00\end{array}$ & $\begin{array}{c}17 \\
25.00\end{array}$ \\
\hline ¿Crees que las redes fomentan las habilidades sociales?? & $\begin{array}{l}N \\
\%\end{array}$ & $\begin{array}{l}0 \\
0\end{array}$ & $\begin{array}{c}4 \\
5.88\end{array}$ & $\begin{array}{c}17 \\
25.00\end{array}$ & $\begin{array}{c}19 \\
27.94\end{array}$ & $\begin{array}{c}28 \\
41.18\end{array}$ \\
\hline ¿El uso de estas redes han mejorado tus habilidades sociales? & $\begin{array}{l}N \\
\%\end{array}$ & $\begin{array}{c}1 \\
1.47\end{array}$ & $\begin{array}{c}6 \\
8.82\end{array}$ & $\begin{array}{c}17 \\
25.00\end{array}$ & $\begin{array}{c}22 \\
32.35\end{array}$ & $\begin{array}{c}22 \\
32.35\end{array}$ \\
\hline
\end{tabular}


Tabla 3

Utilidad de las redes en función del género, edad y ocupación de los participantes. Prueba U de Mann-Whitney.

\begin{tabular}{|c|c|c|c|c|c|c|}
\hline & $\begin{array}{l}\text { ¿Utilizas las redes } \\
\text { para relacionarte con } \\
\text { amigos tradicionales? }\end{array}$ & $\begin{array}{c}\text { ¿Utilizas las redes } \\
\text { para relacionarte con } \\
\text { amigos conocidos de } \\
\text { la red? }\end{array}$ & $\begin{array}{l}\text { ¿Consideras igual } \\
\text { de importantes tus } \\
\text { amigos virtuales que } \\
\text { los tradicionales? }\end{array}$ & $\begin{array}{l}\text { ¿Tienes más amigos } \\
\text { desde que entras a las } \\
\text { redes? }\end{array}$ & $\begin{array}{l}\text { ¿Crees que las } \\
\text { redes fomentan las } \\
\text { habilidades sociales? }\end{array}$ & $\begin{array}{l}\text { ¿El uso de estas redes } \\
\text { ha mejorado tus } \\
\text { habilidades sociales? }\end{array}$ \\
\hline \multicolumn{7}{|l|}{ Género } \\
\hline Mujer & 34.67 & 34.67 & 40.66 & 38.64 & 36.33 & 42.66 \\
\hline Varón & 34.37 & 34.37 & 29.92 & 31.42 & 33.14 & 28.44 \\
\hline$U$ & 560.5 & $335^{*}$ & $387 *$ & 445.5 & 498 & $329^{*}$ \\
\hline$P$ & .949 & .003 & .021 & .113 & .376 & .003 \\
\hline \multicolumn{7}{|l|}{ Edad } \\
\hline$<25$ & 41.87 & 26.84 & 26.02 & 29.08 & 31.44 & 24.68 \\
\hline$>25$ & 28.32 & 40.92 & 41.61 & 39.04 & 37.07 & 42.73 \\
\hline$U$ & $345^{*}$ & $336^{*}$ & $310^{*}$ & $405.5^{*}$ & 541 & $269^{* *}$ \\
\hline$P$ & .004 & .002 & .001 & .027 & .672 & .000 \\
\hline \multicolumn{7}{|l|}{ Ocupación } \\
\hline Estudia & 49.80 & 20.76 & 22.15 & 24.07 & 33.04 & 15.91 \\
\hline Trabaja & 36.31 & 36.04 & 40.08 & 38.19 & 39.08 & 38.88 \\
\hline Sin ocupación & 22.77 & 43.75 & 41.11 & 40.50 & 33.69 & 46.08 \\
\hline$\chi^{2}$ & $26.52^{* * *}$ & $19.9^{* *}$ & $14.82 *$ & $11.14 *$ & 0.98 & $33.53^{* *}$ \\
\hline$P$ & .000 & .000 & .001 & .004 & .612 & .000 \\
\hline
\end{tabular}

Nota: $* p<.05 ; * * p<.001$.

entran a las redes, $U(1)=445.5, p=.113$, ni en el ítem referente a la percepción de que las redes sociales fomentan las habilidades sociales, $U(1)=498, p=.376$, aunque en la siguiente pregunta, con el uso de estas redes han mejorado las habilidades sociales, de nuevo fueron las mujeres las que indicaron estar más de acuerdo en afirmar esta pregunta, $U(1)=329, p<.05$.

En referencia a la utilidad de las redes online según la edad nos encontramos en primer lugar que los más jóvenes las utilizan en mayor medida para relacionarse con los amigos tradicionales, $U(1)=345, p<.05$ mientras que los más mayores para relacionarse con los amigos de la red, $U(1)=336, p<.05$.

De la misma manera, los más mayores indicaron estar más de acuerdo en considerar igual de importantes los amigos virtuales que los tradicionales, $U(1)=41.61, p<.05$, así como en afirmar que tienen más amigos desde que frecuentan estos espacios, $U(1)=405.5, p<.05$.

Por último no encontramos diferencias estadísticamente significativas con respecto a la valoración que hacen sobre las redes como estrategia para fomentar las habilidades sociales, $U(1)=541, p=.672$, aunque si mostraron los jóvenes de más edad que les había ayudado el uso de estos espacios a mejorar sus habilidades sociales, $U(1)=269, p<.001$.

En cuanto a la utilidad de las redes según la ocupación, encontramos que los jóvenes con discapacidad que estudian, seguidos de los que trabajan utilizan más las redes para comunicarse con los amigos tradicionales, $\chi^{2}(2)=6.52, p<.001$, mientras que los jóvenes con discapacidad que indicaron no tener ocupación y los que están trabajando destacaron más en afirmar que utilizan más la red social para relacionarse con amigos virtuales, $\chi^{2}(2)=19.9, p<.001$.

Esta misma dirección muestran las preguntas referentes a la valoración que hacen de los amigos tradicionales y los conocidos a través de la red, en la que indicaron estar más de acuerdo los jóvenes sin ocupación y los jóvenes en situación laboral activa, $\chi^{2}(2)=14.82, p<.05$, así como en la cuestión relativa a si tienen más amigos desde que entran a las redes, en la que observamos que los participantes que no indicaron su ocupación y los que están trabajando mostraron mayor acuerdo con esto, $\chi^{2}(2)=11.14, p<.05$.

Al examinar la percepción que mostraron sobre la utilidad de las redes para fomentar sus habilidades sociales no encontramos diferencias estadísticamente significativas en función de la ocupación, $\chi^{2}(2)=0.98, p=.612$, aunque si observamos que los jóvenes con discapacidad motora que indicaron no tener ocupación y los que están trabajando destacaron más en afirmar que a través de las redes han potenciado sus habilidades sociales, $\chi^{2}(2)=33.53, p<.001$.

\section{Discusión}

En el presente trabajo se pretende reflejar la importancia que tiene para los jóvenes con discapacidad motora las redes sociales online en sus relaciones sociales. Así, tal y como se comprueba en nuestros resultados, podemos observar que este recurso es utilizado por la mayoría de jóvenes, y no tan jóvenes, que tienen una discapacidad motora, frecuentando gran parte de ellos, estos espacios con asiduidad y dedicándole bastante tiempo al día.

Estos resultados reflejan la misma dirección que los datos sobre el uso y preferencia hacia las redes sociales que tienen los jóvenes sin discapacidad, sin embargo, y aunque es evidente que estos espacios son utilizados por usuarios sin ningún tipo de discapacidad, para algunos jóvenes con características específicas, como vivir con movilidad reducida puede significar un recurso especialmente útil a la hora de aportarles la posibilidad de reducir sus limitaciones e incrementar las vías de interacción. Esto explicaría el hecho de que en nuestros resultados 
encontremos que la mayoría esté bastante de acuerdo en relacionarse con amigos que conocen a través de la red.

En referencia a esto, anteriormente se mencionó que las personas con discapacidad en muchas ocasiones ven reducido su grupo social debido a las limitaciones derivadas de su discapacidad y esto, puede desembocar en un mayor nivel de aislamiento, por tanto, a través de internet, encuentren una vía para acceder a las relaciones sociales con más facilidad que de forma tradicional.

Esto, podría inducirnos a pensar que los jóvenes utilizan estos espacios como última alternativa para relacionarse ya que no tienen relaciones con amigos cara a cara y con ello, a pensar que estas relaciones sean por "recurso" o por "conveniencia" al carecer de relaciones tradicionales. Aun pudiendo esto ser cierto, no significa que estas relaciones sean superficiales puesto que al examinar la opinión sobre la calidad de las relaciones mantenidas por las redes sociales online se comprueba que los participantes consideran que, independientemente de que interaccionen con amigos de la vida real o que sean amigos conocidos por vía online, la amistad es valiosa y en absoluto trivial. Esto sigue la misma dirección que la apuntada por algunos autores (Mickelson, 1997). Algunos usuarios se sienten mucho más cómodos por internet afirmando que este medio les ofrece unas ventajas que no obtienen en las relaciones cara a cara.

De esta forma, ello sugiere que muchos de los usuarios encuentran en estas redes unos espacios ideales para relacionarse y mantener vínculos profundos. Nuestros resultados también van en contra de la idea de que los vínculos establecidos vía online son de lazo débil (Adelman, Parks y Albrecht, 1987).

Con respecto a los ítems del cuestionario en función del género, observamos que las mujeres con discapacidad motora conectan, tienen más amistades a través de internet y están más de acuerdo en que a través de las redes online han mejorado sus habilidades sociales.

Posiblemente, por desgracia, los estereotipos y modas que marca la sociedad alejan mucho a las personas con discapacidad física de los cánones de lo atractivo, esto sobre todo, se potencia más en la mujer. Por ejemplo, en toda situación "cara a cara" están presentes de manera implícita determinados condicionantes sociales acerca de cómo "deben ser" las personas. Ciertos ideales estéticos determinan, en cierto modo, como debe ser nuestro aspecto. Esto especialmente afecta a las mujeres de edades jóvenes. Sin embargo, esto desaparece en el ciberespacio donde todo se limita a lo escrito y no existe la presencia (física) del otro. Al no existir la influencia que proporciona la imagen física, podemos establecer una relación con el otro sin verlo y escribir sobre nosotros mismos sin que nuestra conversación quede marcada por nuestro aspecto (Hartley, 2002), actuando la red como un espacio liberador en el que poder expresarse sin temor a ser juzgada la imagen.

Al examinar si influye la edad nos encontramos que el grupo de mayor edad utiliza más las redes para relacionarse con amigos exclusivamente de la red, mientras que los más jóve- nes, lo hacen en mayor medida para mantener contacto con sus amigos de su grupo social tradicional. Esto podría explicarse por el tipo de actividades que realizan habitualmente. Es lógico suponer que los más jóvenes están vinculados a contextos educativos en los que forzosamente tienen que relacionarse con amigos o compañeros de clase. Sin embargo, conforme avanza la edad, es más habitual realizar otras actividades desvinculadas del mundo académico con ello, no ser necesario tener que mantener un contacto tan frecuente con los compañeros.

Esta idea podría quedar respaldada al observar los resultados relacionados con la opinión y tipo de relación que mantienen los participantes en función de la ocupación. Por ejemplo, hemos encontrado que los que estudian utilizan más estos espacios para comunicarse con sus amigos tradicionales mientras que los usuarios que trabajan, se conectan para relacionarse con amigos virtuales.

En cuanto a la valoración que hacen los jóvenes con discapacidad sobre si mejoran las habilidades sociales, así como si a ellos, les ha ayudado a potenciar esta cualidad los resultados muestran que la mayoría indica estar muy de acuerdo, siendo los más mayores los que más a favor están con esta afirmación.

Estos resultados siguen la misma dirección que la de otros autores que indican que el uso de internet como recurso para relacionarse no sólo mejora la interacción y la existencia de relaciones sociales, sino que incluso aumenta la cantidad de conexiones en la red (Fowler y Christakis, 2009; Zubeidat et al., 2007).

Aunque no cabe duda del potencial de las redes online como fuente de relaciones interpersonales para los jóvenes y en especial para los jóvenes con limitaciones físicas como es tener una discapacidad motora, así como del interés de los resultados obtenidos, el trabajo presenta algunas limitaciones que es necesario considerar.

En primer lugar, el número de participantes es muy reducido ni el muestreo fue probabilístico, sino que fue por conveniencia. En este sentido, se utilizó el muestreo "bola de nieve", así, inicialmente se colocó el enlace en varias direcciones de diferentes redes sociales, transcendiendo el enlace a usuarios que, aunque eran conocidos por los usuarios con los que contactamos en un principio, desconocemos sus características.

Igualmente, debemos ser cautos y considerar algunas limitaciones del estudio relacionadas con el instrumento para recoger la información y con la deseabilidad social. Así, nuestros participantes son usuarios y al realizar un autoinforme sobre las cuestiones referidas en el cuestionario pueden estar sesgando sus respuestas.

Por tanto, sería interesante superar estas limitaciones y ampliar el estudio a una muestra más elevada y representativa de jóvenes con discapacidad motora. Del mismo modo sería interesante comparar la utilización de las redes y la percepción que tienen de estos espacios para mejorar sus relaciones con jóvenes con distinta tipología de discapacidad así como con jóvenes que no padecen esta problemática. 


\section{Referencias}

1. Adelman, M., Parks, M. y Albrecht, T. (1987). Beyond close relationships: Support in weak ties. En T. L. Albrecht y M. B. Adelman (Eds.), Communicating Social Support (pp.126-147). Newbury Park, CA: Sage.

2. Aguaded, J.I. (2009). El Parlamento Europeo apuesta por la alfabetización mediática. Comunicar, 32, 7-8.

3. Alexander, S. C., Peterson, J. L. y Hollingshead, A. B. (2003). Help is at your keyboard: support groups on the Internet. In L. R. Frey (Ed.), Group communication in context: Studies of bona fide groups (pp. 309-334). New York: Erlbaum.

4. Asociación para la Investigación de los Medios de Comunicación. (2011). Navegantes en la red. 13 ${ }^{\mathrm{a}}$ encuesta AIMC a usuarios de Internet (http://www.aimc.es)

5. Beranuy, M., Lusar, A. Graner, C. y Carbonell Sánchez, X. (2009). Validación de dos escalas breves para evaluar la adicción a Internet y el abuso del móvil. Psicothema, 21, 480-485.

6. Bernete, F. (2010). Usos de las TIC, relaciones sociales y cambios en la socialización de las y los jóvenes. Revista de estudios de juventud, 88, 97-114.

7. Buckingham, D. (2008). Más allá de la tecnología. Aprendizaje infantil en la era de la cultura digital. Buenos Aires: Manantial.

8. Cruzado, L., Matos, L. y Kendall, R. (2006). Adicción a Internet: Perfil clínico y epidemiológico de pacientes hospitalizados en un instituto nacional de salud mental. Revista Medica Herediana, 17, 196-205.

9. Echeburúa, E. y Corral, P. (2009). Las adicciones con o sin droga: una patología de la libertad. En E. Echeburúa, F.J. Labrador y E. Becoña (Eds.), Adicción a las nuevas tecnologías en adolescentes y jóvenes (pp. 29-44). Madrid: Pirámide.

10. Finn, J. (1999). An exploration of helping processes in an online self-help group focusing on issues of disability. Health and Social Work, 24, 220-231. http://dx.doi. org/10.1093/hsw/24.3.220

11. Fogel J., Albert S.M., Schnabel F., Ditkoff, B.A. y Neugut, A. I. (2002). Internet use and social support in women with breast cancer. Health Psychology, 21, 398-404. http:// dx.doi.org/10.1037/0278-6133.21.4.398

12. Fowler, J.H. y Christakis, N.A. (2009). The Dynamic Spread of Happiness in a Large Social Network: Longitudinal Analysis Over 20 Years in the Framingham Heart Study. British Medical Journal, 337, 1-9.

13. García del Castillo, J.A., Terol, M.C., Nieto, M., Lledó, A., Sánchez, S., Martín-Aragón, M. y Sitges, E. (2008). Uso y abuso de Internet en jóvenes universitarios. Adicciones, 20, 131-142.

14. Granovetter, M. S. (1973). The strength of weak ties. American Journal of Sociology, 78, 1360-1380. http://dx.doi. org/10.1086/225469
15. Hartley, J. (2002). Communication, Cultural and Media Studies: The Key Concepts. London: Routledge. http:// dx.doi.org/10.4324/9780203449936

16. Hoybye, M. T., Johansen, C., y Tjornhoj-Thomsen, T. (2005). Online interaction: Effects of storytelling in an Internet breast cancer support group. Psycho-Oncology, 14, 211-220. http://dx.doi.org/10.1002/pon.837

17. INE (2008). Encuesta de Discapacidad, Autonomía personal y situaciones de Dependencia. Madrid: EDAD.

18. Jimenez, L. y Pantoja, A. V. (2007). Autoestima y relaciones Interpersonales en sujetos adictos a Internet. Psicología-Segunda Época, 26, 78- 89.

19. Kraut, R., Patterson, M., Landmark, V., Kiesler, S., Mukophadhyay, T. y Scherlis, W. (1998). Internet paradox: a social technology that reduces social involvement and psychological well being? American Psychologist, 53, 10171031. http://dx.doi.org/10.1037/0003-066X.53.9.1017

20. Latiesa, M. (1996).Validez y fiabilidad de las observaciones sociológicas. En M. García, J. Ibáñez y F.Alvira (Eds.), El análisis de la realidad social. Métodos y técnicas de investigación. Madrid: Alianza.

21. Lenhart, A., Madden, M., Rankin Macgill, A. y Smith, A. (2007). Teens and social media: The use of social media gains a greater foothold in teen life as they embrace the conversational nature of interactive online media. Washington, DC: Pew Internet \& American Life Project. Obtenido el 10 de septiembre del 2011. En: http://www.pewinternet.org//media//Files/Reports/2007/PIP_Teens_Social_ Media Final.pdf.pdf

22. Livingstone, S. (2008). Taking risky opportunities in youthful content creation: teenagers' use of social networking sites for intimacy, privacy and self-expression. New media and society, 10, 393-411. http://dx.doi. org/10.1177/1461444808089415

23. Livingstone, S. y Helsper, E. (2010). Balancing Opportunities and Risks in Teenagers' Use of the Internet: the Role of On-line Skills and Internet Self-efficacy. New Media \& Society, 12, 309-329. http://dx.doi. org/10.1177/1461444809342697

24. Losada, J. L. y López-Feal, R. (2003). Métodos de investigación en ciencias humanas y sociales. Madrid: Thomson.

25. Mickelson, K. D. (1997). Seeking social support: Parents in electronic support groups. En S. Kiesler (Ed.), Culture of the Internet (pp. 157-178). Mahwah, NJ: Lawrence Erlbaum.

26. Morahan-Martin, J. y Schumacher, P. (2003). Loneliness and social uses of the Internet. Computers in Human Behavior, 19, 659-671. http://dx.doi.org/10.1016/S07475632(03)00040-2

27. Moral, J.A. de (2007). Redes sociales y wikis. En O. Rojas, J.L. Antúnez, J.A. Gelado, J.A. del Moral, J.A. Casas y R. Alatriste (Eds.), Web 2.0. Madrid: ESIC.

28. Niemz, K., Griffiths, M. y Banyard, P. (2005). Prevalence 
of pathological Internet use among university students and correlations with self-esteem, the general health qustionaire (GHQ), and disinhibition. CyberPsychology \& Behavior, 8, 562-570. http://dx.doi.org/10.1089/cpb.2005.8.562

29. Poch, F. (2009). Uso autoinformado de Internet en adolescentes: Perfil psicológico de un uso elevado de red. International Journal of Psychology \& Psychological Therapy, 9, 109-122.

30. Robinson, J., Kestnbaum, M., Neustadtl, A. y Alvarez, A. (2000). Mass Media and social life among internet users. Social Science Computer Review, 18, 490-501. http://dx.doi.org/10.1177/089443930001800411

31. Rubio-Gil, A. (2010). Generación digital: patrones de consumo de internet, cultura juvenil y cambio social. Revista de estudios de juventud, 88, 201-221.

32. Suriá, R. y Beléndez, M. (2011). Grupos de apoyo virtuales dedicados a problemas de salud: estudio de su tipología y análisis de su representatividad. Anales de Psicología, 27, 210-220.

33. Tully, C.J. (2007). La socialización en el presente digital. Informalización y contextualización. Revista iberoamericana de ciencia, tecnología y sociedad, 8, 9-22.

34. UCLA Internet Report (2000). Surveying the Digital Future. Los Angeles: UCLA. Obtenido el 12 de noviembre de 2005. En: http://www.ccp.ucla.edu/newsite/pages/ internet-report.asp.
35. Weinberg, N., Schmale, J., Uken, J. y Wessel, K. (1995). Computer-mediated support groups. Social Work with Groups, 17, 43-54. http://dx.doi.org/10.1300/ J009v17n04 04

36. Wright, K. (2000). Computer-mediated social support, older adults, and coping. Journal of Communication, 50, 100-118. http://dx.doi.org/10.1111/j.1460-2466.2000. tb02855.x

37. Yang, S.C. y Tung, C. (2007). Comparison of Internet Addicts and non-Addicts in Taiwanese High School. Computers in Human Behavior, 23, 79-96. http://dx.doi. org/10.1016/j.chb.2004.03.037

38. Young K. (2009). Online Social Networking: An Australian Perspective. International Journal of Emerging Technologies \& Society, 7, 39-57.

39. Zubeidat, I., Salinas, J. y Sierra, J. (2008). Evaluacion de factores asociados a la ansiedad social y a otras psicopatologias en adolescentes, Clinica y salud, 31, 189-196.

Fecha de recepción: 16 de junio de 2012

Fecha de recepción de la versión modificada: 1 de septiembre de 2012 Fecha de aceptación: 18 de septiembre de 2012 